\title{
Periodic Solutions to Second-Order Nonlinear Differential Equations in Banach Spaces
}

\author{
David Ariza-Ruiz@ and Jesús Garcia-Falset
}

\begin{abstract}
In this article, we deal with the existence of solutions for the following second-order differential equation:

$$
\left\{\begin{array}{l}
u^{\prime \prime}(t)=f(t, u(t))+h(t) \\
u(a)-u(b)=u^{\prime}(a)-u^{\prime}(b)=0,
\end{array}\right.
$$

where $\mathbb{B}$ is a reflexive real Banach space, $f:[a, b] \times \mathbb{B} \rightarrow \mathbb{B}$ is a sequentially weak-strong continuous mapping, and $h:[a, b] \rightarrow \mathbb{B}$ is a continuous function on $\mathbb{B}$. The proofs are obtained using a recent generalization of the well-known Bolzano-Poincaré-Miranda theorem to infinite-dimensional Banach spaces. In the last section, we present three examples of application of the general result.
\end{abstract}

Mathematics Subject Classification. Primary 34A34; Secondary 34G05.

Keywords. Second-order differential equations, Periodic solutions.

\section{Introduction}

The main purpose of this article is to study the existence of solutions of the second-order dynamical equation

$$
\left\{\begin{array}{l}
u^{\prime \prime}(t)=f(t, u(t))+h(t) \\
u(a)-u(b)=u^{\prime}(a)-u^{\prime}(b)=0,
\end{array}\right.
$$

where $\mathbb{B}$ is a reflexive real Banach space, $f:[a, b] \times \mathbb{B} \rightarrow \mathbb{B}$ is a continuous mapping, and $h:[a, b] \rightarrow \mathbb{B}$ is a continuous function on $\mathbb{B}$. During the previous decades, some classical tools have been used in the study of periodic solutions of Eq. (1.1) in finite-dimensional Banach spaces, including the method of upper and lower solutions $[6,10,13]$, and degree theory and fixed point theory $[8,12]$. In this sense, in [20], the author gave a generalization of MirandaPoincaré theorem and using this generalization proved theorems about the 
existence for systems of $k$ equations $x^{\prime \prime}=f\left(t, x, x^{\prime}\right)$, where $f:[0,1] \times \mathbb{R}^{k} \times$ $\mathbb{R}^{k} \rightarrow \mathbb{R}^{k}$ is a vector function, subject to various boundary conditions.

Recently, the existence of periodic solutions of second-order differential equations on a general Banach space has been treated in several papers; for instance, see $[14,17,19]$. In [14], operator-valued Fourier multiplier theorems are used to study this problem. In [17], the authors developed an infinitedimensional version of Poincaré-Miranda theorem and they showed their relation with viability theory for differential inclusions and how to apply them, in particular, in the context of constrained PDEs. On the other hand, in [19], variational methods are used to study the existence of two solutions of superlinear second-order Hamiltonian systems that have damped and impulsive effects.

In this paper, we are able to give sufficient conditions to obtain existence of periodic solutions for Problem (1.1). Namely, we obtain that when $\mathbb{B}$ is a reflexive Banach space and $f:[a, b] \times \mathbb{B} \rightarrow \mathbb{B}$ is a sequentially weak-strong continuous mapping satisfying conditions (3.2) and (3.4) (see them below), then, using a generalization of Bolzano-Poincaré-Miranda theorem [1] and Leray-Schauder Alternative theorem (for instance, see [4]), Problem (1.1) admits a classical solution.

\section{Notations and Preliminaries}

In this section, we shall recall some definitions and results that are needed later on.

Throughout this article, $\mathbb{B}$ will denote a real reflexive Banach space with norm $\|\cdot\|$ and we shall denote its dual by $\mathbb{B}^{*}$. The closed ball of center $x_{0}$ and radius $r$ is denoted by $B_{r}\left[x_{0}\right]$ and the sphere of center $x_{0}$ and radius $r$ by $\partial B_{r}\left[x_{0}\right]$. The symbol " $\rightarrow$ " is used to denote the convergence of a sequence in $\mathbb{B}$ with respect to the weak topology $\sigma\left(\mathbb{B}, \mathbb{B}^{*}\right)$, as well as the symbol " $\rightarrow$ " is used to denote the convergence of a sequence in $\mathbb{B}$ with respect to the norm topology.

Recall that the normalized duality mapping $J: \mathbb{B} \rightarrow 2^{\mathbb{B}^{*}}$ is defined by

$$
J(x):=\left\{j \in \mathbb{B}^{*}:\langle x, j\rangle=\|x\|^{2},\|j\|=\|x\|\right\}, \quad x \in \mathbb{B} .
$$

Let $\langle y, x\rangle_{+}:=\max \{\langle y, j\rangle: j \in J(x)\}$ and let $\langle y, x\rangle_{-}:=\min \{\langle y, j\rangle: j \in$ $J(x)\}$. For more details, see [5].

Denote by $\mathcal{C}([a, b], \mathbb{B})$ the space of $\mathbb{B}$-valued continuous functions on $[a, b]$ with the norm $\|u\|_{\infty}=\sup \{\|u(t)\|: t \in[a, b]\}$, and denote by $L^{1}(a, b, \mathbb{B})$ the space of $\mathbb{B}$-valued Bochner integrable functions on $[a, b]$ with the norm $\|u\|_{1}=$ $\int_{a}^{b}\|u(t)\| \mathrm{d} t$. It is well known (for example, see [18]) that, if $u \in \mathcal{C}([a, b], \mathbb{B})$, then $u \in L^{1}(a, b, \mathbb{B})$ and, for each $t \in[a, b]$, we have that $\frac{\mathrm{d}}{\mathrm{d} t}\left(\int_{a}^{t} u(s) \mathrm{d} s\right)=u(t)$.

Let $K$ be a nonempty subset of $\mathbb{B}$. A mapping $F: K \rightarrow \mathbb{B}$ is said to be compact if $F(C)$ is relatively compact whenever $C$ is a bounded subset of $K$. If, moreover, $F$ is continuous in $K$, then $F$ is called completely continuous. Clearly, every continuous mapping with closed domain in a finite-dimensional Banach space is completely continuous. On the other hand, $F$ is said to be 
sequentially weak-strong continuous mapping if given any sequence $\left(x_{n}\right)$ in $K$, such that $x_{n} \rightarrow x \in K$ the strong convergence $F\left(x_{n}\right) \rightarrow F(x)$ holds. It is clear that if $\mathbb{B}$ is a reflexive Banach space, $K$ is a closed convex subset of $\mathbb{B}$, and $F: K \rightarrow \mathbb{B}$ is a sequentially weak-strong continuous mapping, then $F$ is completely continuous.

Theorem 2.1 (Schauder's Fixed Point Theorem). Let $K$ be a nonempty, bounded, closed, and convex subset of a Banach space $\mathbb{B}$. If $F: K \rightarrow K$ is completely continuous, then $F$ has a fixed point.

The hypothesis that is used to produce a more convenient form of Schauder's fixed point theorem is called Leray-Schauder boundary condition: Given a mapping $F: \mathbb{B} \rightarrow \mathbb{B}$, the mapping $F$ satisfies this condition if there exists $r>0$, such that $\|x\|=r$ implies $F(x) \neq \lambda x$ for all $\lambda>1$. The result that replaces the requirement in Schauder's fixed point theorem is the following one (see, e.g., [4]):

Theorem 2.2 (Leray-Schauder Alternative). Let $\mathbb{B}$ be a Banach space. If $F: \mathbb{B} \rightarrow \mathbb{B}$ is a completely continuous mapping and $F$ satisfies the LeraySchauder condition, then $F$ has a fixed point.

We state below Ascoli-Arzelà's theorem (see [7, p.34]) exactly in the form we need later as follows:

Theorem 2.3 (Ascoli-Arzelà). Let $(\mathbb{B},\|\cdot\|)$ be a Banach space and let $K \subseteq$ $\mathcal{C}([a, b], \mathbb{B})$ be a family of functions, such that

(i) $\|u(t)\| \leq M$, for all $t \in[a, b], u \in K$.

(ii) $K$ is equi-uniformly continuous, i.e., for all $\epsilon>0$, there exists $\delta(\epsilon)>0$, such that $\|u(t)-u(s)\| \leq \epsilon$, if $|t-s| \leq \delta(\epsilon)$, for all $u \in K$.

(iii) For each $t \in[a, b]$, the set $\{u(t): u \in K\}$ is compact in $\mathbb{B}$.

Then, $K$ is compact in $\mathcal{C}([a, b], \mathbb{B})$.

Recently, in [1, Theorem 3.1], an extension of the well-known BolzanoPoincaré-Miranda theorem to infinite-dimensional Banach spaces is obtained. A particular version of this result, which we will use, is the following one (see Remark 3.1 and Corollary 3.1 in [1]).

Proposition 2.1. Given a reflexive Banach space $\mathbb{B}$, a point $z \in \mathbb{B}$, and a number $R>0$, let $F: B_{R}[z] \rightarrow \mathbb{B}$ be a weak-strong continuous mapping. Assume that there exists a functional $[\cdot, \cdot]_{c}: \mathbb{B} \times \mathbb{B} \rightarrow \mathbb{R}$ satisfying

$\left(C_{1}\right)[x, x]_{c}>0$, for all $x \in \mathbb{B}$ with $x \neq 0$;

$\left(C_{2}\right)[\lambda x, x]_{c}=\lambda[x, x]_{c}$, for all $x \in \mathbb{B}$ and $\lambda \in \mathbb{R}$.

If $[F(x), x-z]_{c}$ has constant sign for all $x \in \partial B_{R}[z]$, then $F$ has at least $a$ zero in $B_{R}[z]$.

We finish this section recalling several basic definitions and relevant results related to Sobolev spaces.

Let $\Omega$ be a measurable subset of $\mathbb{R}^{N}$ which for simplicity will be assumed to be bounded. The Sobolev space $W^{m, p}(\Omega)$ (see $\left.[3,18]\right)$ is the Banach space 
of all functions in $L^{p}(\Omega)$ whose weak derivatives up to order $m$ also belong to $L^{p}(\Omega)$. The norm in $W^{m, p}(\Omega)$ is given by

$$
\|u\|_{m, p}=\|u\|_{p}+\sum_{1 \leq|\alpha| \leq m}\left\|D^{\alpha} u\right\|_{p}
$$

or by the equivalent norm

$$
\|u\|_{m, p}=\left(\|u\|_{p}^{p}+\sum_{1 \leq|\alpha| \leq m}\left\|D^{\alpha} u\right\|_{p}^{p}\right)^{\frac{1}{p}}
$$

where $\alpha=\left(\alpha_{1}, \ldots, \alpha_{N}\right) \in \mathbb{R}^{N}$ is a multi-index of non-negative integers with order $|\alpha|=\sum_{i=1}^{N} \alpha_{i}$ and $D^{\alpha} u=\frac{\partial^{|\alpha|}}{\partial x_{1}^{\alpha} \ldots \partial x_{N}^{\alpha_{N}}} u$. $W_{0}^{m, p}(\Omega)$ is the closure of $\mathcal{C}_{c}^{\infty}(\Omega)$ in $W^{m, p}(\Omega)$. Here, $\mathcal{C}_{c}^{\infty}(\Omega)$ denotes the space of infinitely differentiable functions $\phi: \Omega \rightarrow \mathbb{R}$ with compact support in $\Omega$.

The next inequality is known as "Poincaré's inequality" and it is very useful in the study of Dirichlet elliptic equations (for instance, see $[18$, Section 13.2]) as follows:

Proposition 2.2. There exists a positive constant $\boldsymbol{c}=\boldsymbol{c}(\Omega, p)$, such that

$$
\|w\|_{p} \leq \boldsymbol{c}\|\nabla w\|_{p}
$$

for all $w \in W_{0}^{1, p}(\Omega)$.

As a consequence of the above inequality, we get to $\|\nabla u\|_{2}$ is an equivalent norm on $W_{0}^{1,2}(\Omega)$, namely

$$
\frac{1}{\sqrt{c^{2}+1}}\|w\|_{1,2} \leq\|\nabla w\|_{2} \leq\|w\|_{1,2}, \quad \text { for all } w \in W_{0}^{1,2}(\Omega)
$$

where we are considering that $\|w\|_{1,2}=\sqrt{\|w\|_{2}^{2}+\|\nabla w\|_{2}^{2}}$, and $\|\nabla w\|_{2}=$ $\sqrt{\sum_{i=1}^{n}\left\|\frac{\partial w}{\partial x_{i}}\right\|_{2}^{2}}$.

\section{Main Results}

In 1971, Knobloch [16] showed that if $f:[0, T] \times \mathbb{R}^{n} \rightarrow \mathbb{R}^{n}$ is a continuous function which is locally Lipschitzian in $u$, the condition: there exists $R>0$, such that $\langle f(t, u), u\rangle \geq 0$ for all $t \in[0, T]$ and $u \in \mathbb{R}^{n}$ satisfying $\|u\|=R$ is a sufficient condition for the existence of a solution for Problem (1.1). In our case, we have to work with conditions (3.2) and (3.4) (see below).

For our subsequent study, we introduce, for each element of the Banach space $\mathcal{C}([a, b], \mathbb{B})$, an auxiliary mapping and we will prove the existence and uniqueness of zeros of this mapping. Let $v \in \mathcal{C}([a, b], \mathbb{B})$. We define $F_{v}: \mathbb{B} \rightarrow \mathbb{B}$ as

$$
F_{v}(x)=\int_{a}^{b} f\left(\tau, \int_{a}^{\tau} v(s) \mathrm{d} s+x\right) \mathrm{d} \tau
$$


Lemma 3.1. Let $v \in \mathcal{C}([a, b], \mathbb{B})$ and let $f:[a, b] \times \mathbb{B} \rightarrow \mathbb{B}$ be a sequentially weak-strong continuous function satisfying that

for each $M>0$, there exists $\delta_{M}>0$, such that $\langle f(t, y+x), x\rangle_{-} \geq 0$,

for all $t \in[a, b], y \in B_{M}[0]$ and $\|x\| \geq \delta_{M}$.

Then, the mapping $F_{v}: \mathbb{B} \rightarrow \mathbb{B}$ given by (3.1) has at least a zero in $\mathbb{B}$.

Proof. Let $v \in \mathcal{C}([a, b], \mathbb{B})$. First, we are going to see that $F_{v}$ is sequentially weak-strong continuous. Let $\left\{x_{n}\right\}$ be a bounded sequence in $\mathbb{B}$, i.e., there exists $r>0$, such that $x_{n} \in B_{r}[0]$ for all $n \in \mathbb{N}$. Since $\mathbb{B}$ is reflexive, there exists a weakly convergent subsequence $\left\{x_{n_{k}}\right\}$ of $\left\{x_{n}\right\}$, say $x_{n_{k}} \rightarrow x \in B_{r}[0]$. We consider the sequence of functions given by $g_{n_{k}}(t):=f\left(t, \int_{a}^{t} v(s) \mathrm{d} s+x_{n_{k}}\right)$, for $t \in[a, b]$. By hypothesis, $f$ is sequentially weak-strong continuous; then, for each $t \in[a, b], g_{n_{k}}(t)$ converges strongly to $g(t):=f\left(t, \int_{a}^{t} v(s) \mathrm{d} s+x\right)$. Furthermore, for each $n_{k}$, we get to $\left\|g_{n_{k}}(t)\right\| \leq K$ for all $t \in[a, b]$, where $K \geq 0$ is the constant of boundedness of $f\left([a, b] \times B_{r+(b-a)\|v\|_{\infty}}[0]\right)$. Thus, for each $k \in \mathbb{N}$

$$
\begin{aligned}
\left\|F_{v}\left(x_{n_{k}}\right)-F_{v}(x)\right\| & \leq \int_{a}^{b}\left\|f\left(t, \int_{a}^{t} v(s) \mathrm{d} s+x_{n_{k}}\right)-f\left(t, \int_{a}^{t} v(s) \mathrm{d} s+x\right)\right\| \mathrm{d} t \\
& =\int_{a}^{b}\left\|g_{n_{k}}(t)-g(t)\right\| \mathrm{d} t,
\end{aligned}
$$

which implies, by the dominated convergence theorem, the strong convergence of $\left\{F_{v}\left(x_{n_{k}}\right)\right\}$ to $F_{v}(x)$.

On the other hand, if we consider the functional $[\cdot, \cdot]_{c}: \mathbb{B} \times \mathbb{B} \rightarrow \mathbb{R}$ given by

$$
[x, y]_{c}=\langle x, y\rangle_{+}:=\sup _{j \in J(y)}\langle x, j\rangle=\max _{j \in J(y)}\langle x, j\rangle,
$$

then $[\cdot, \cdot]_{c}$ satisfies $\left(C_{1}\right)-\left(C_{2}\right)$. Moreover, taking $M:=(b-a)\|v\|_{\infty}$, we know that, for all $\tau \in[a, b], \int_{a}^{\tau} v(s) \mathrm{d} s \in B_{M}[0]$. By (3.2), there exists $\delta_{M}>0$, such that $\left\langle f\left(t, \int_{a}^{t} v(s) \mathrm{d} s+x\right), x\right\rangle_{-} \geq 0$, for all $t \in[a, b]$ and $\|x\| \geq \delta_{M}$. Therefore, for all $x \in \partial B_{\delta_{M}}[0]$, we have that

$$
\left[F_{v}(x), x\right]_{c}=\left\langle F_{v}(x), x\right\rangle_{+} \geq \int_{a}^{b}\left\langle f\left(\tau, \int_{a}^{\tau} v(s) \mathrm{d} s+x\right), x\right\rangle_{-} \mathrm{d} \tau \geq 0 .
$$

Applying Proposition 2.1, we deduce that $F_{v}$ has at least a zero in $B_{\delta_{M}}[0]$.

Remark 3.1. Bearing in mind Proposition 2.1 and the proof of Lemma 3.1, in Lemma 3.1, we can, clearly, replace Condition (3.2) by

for each $M>0$ there exists $\delta_{M}>0$ such that $\langle f(t, y+x), x\rangle_{+} \leq 0$

for all $t \in[a, b], y \in B_{M}[0]$ and $\|x\| \geq \delta_{M}$.

Lemma 3.2. Let $v \in \mathcal{C}([a, b], \mathbb{B})$ and let $f:[a, b] \times \mathbb{B} \rightarrow \mathbb{B}$ be a strictly accretive operator with respect to the second variable, that is

$$
\langle f(t, x)-f(t, y), x-y\rangle_{-}>0 \text { for all } t \in[a, b], x, y \in \mathbb{B}, \text { with } x \neq y \text {. }
$$


Then, if there is a zero of the operator $F_{v}: \mathbb{B} \rightarrow \mathbb{B}$, given by (3.1), it is unique.

Proof. Note that, by (3.4), $F_{v}$ is strictly accretive, that is, $\left.\left\langle F_{v}(x)-F_{v}(y), j\right\rangle\right\rangle$ 0 for all $x, y \in \mathbb{B}$, with $x \neq y$ and for all $j \in J(x-y)$. Indeed, for each $x, y \in \mathbb{B}$ and $j \in J(x-y)$, we have that

$$
\begin{aligned}
\left\langle F_{v}(x)-F_{v}(y), j\right\rangle & =\left\langle\int_{a}^{b}\left(f\left(\tau, \int_{a}^{\tau} v(s) \mathrm{d} s+x\right)-f\left(\tau, \int_{a}^{\tau} v(s) \mathrm{d} s+y\right)\right) \mathrm{d} \tau, j\right\rangle \\
& =\int_{a}^{b}\left\langle f\left(\tau, \int_{a}^{\tau} v(s) \mathrm{d} s+x\right)-f\left(\tau, \int_{a}^{\tau} v(s) \mathrm{d} s+y\right), j\right\rangle \mathrm{d} \tau .
\end{aligned}
$$

Since $j \in J(x-y)=J\left(x+\int_{a}^{\tau} v(s) \mathrm{d} s-\left(y+\int_{a}^{\tau} v(s) \mathrm{d} s\right)\right)$, from (3.4), we obtain that $\left\langle F_{v}(x)-F_{v}(y), j\right\rangle>0$ for all $x, y \in \mathbb{B}$, with $x \neq y$ and for all $j \in J(x-y)$.

Now, suppose that $z_{1}, z_{2} \in \mathbb{B}$ are two zeros of $F_{v}$ with $z_{1} \neq z_{2}$. Since $F_{v}$ is strictly monotone, $\left\langle F_{v}\left(z_{1}\right)-F_{v}\left(z_{2}\right), j\right\rangle>0$ for all $j \in J\left(z_{1}-z_{2}\right)$ which contradicts that $F_{v}\left(z_{i}\right)=0, i=1,2$. Therefore, $z_{1}=z_{2}$, that is, if $F_{v}$ has a zero, it is unique.

Remark 3.2. Checking the proof of Lemma 3.2, we can replace the hypothesis of strict accretiveness with respect to the second variable (3.4) by being a strictly dissipative operator, that is

$$
\begin{aligned}
& f:[a, b] \times \mathbb{B} \rightarrow \mathbb{B} \text { saisfies }\langle f(t, x)-f(t, y), x-y\rangle_{+}<0 \\
& \text { for all } t \in[a, b], x, y \in \mathbb{B} \text {, with } x \neq y .
\end{aligned}
$$

At this point, we can prove the main result of this work related to the existence of a periodic solution of Problem (1.1).

Theorem 3.1. Let $f:[a, b] \times \mathbb{B} \rightarrow \mathbb{B}$ be a sequentially weak-strong continuous function satisfying (3.2) and (3.4) (or (3.3) and (3.5), respectively). Let $h:[a, b] \rightarrow \mathbb{B}$ be a continuous function with $\int_{a}^{b} h(t) \mathrm{d} t=0$. Then, Problem (1.1) has at least one solution $u \in \mathcal{C}^{2}([a, b], \mathbb{B})$ whenever one of the following assumptions holds:

$\left(A_{1}\right)$. [A condition of boundedness] $f$ is bounded.

$\left(A_{2}\right)$. [A growth condition] There exists a non-decreasing function $\Gamma$ : $[0, \infty) \rightarrow[0, \infty)$ satisfying

$$
\lim _{s \rightarrow \infty} \frac{\Gamma\left(s+\delta_{s}\right)}{s}<\frac{1}{2(b-a)^{2}},
$$

where $\delta_{s}$ is the constant given in (3.2), such that

$$
\|f(t, x)\| \leq \Gamma(\|x\|), \quad \text { for all }(t, x) \in[a, b] \times \mathbb{B} .
$$

Proof. For the sake of clarity, the proof will be divided into five steps.

Step 1. Notice that if we consider $v(t)=u^{\prime}(t)$, then Problem (1.1) is equivalent to solve the following problem:

$$
\left\{\begin{array}{l}
v^{\prime}(t)=f\left(t, \int_{a}^{t} v(s) \mathrm{d} s+D\right)+h(t) \\
v(a)=v(b), \int_{a}^{b} v(t) \mathrm{d} t=0
\end{array}\right.
$$


where $D$ is any element of $\mathbb{B}$ depending of $v$.

Step 2. By Lemmas 3.1 and 3.2, for each $v \in \mathcal{C}([a, b], \mathbb{B})$, there exists a unique zero $D_{v}$ for $F_{v}$ in $B_{R}[0]$, where $R:=\delta_{M}$ and $M:=(b-a)\|v\|_{\infty}$.

Integrating Eq. (3.6), we deduce that the previous problem is equivalent to the following integral equation:

$$
v(t)=\int_{a}^{t} f\left(\tau, \int_{a}^{\tau} v(s) \mathrm{d} s+D_{v}\right) \mathrm{d} \tau+\int_{a}^{t} h(\tau) \mathrm{d} \tau+v(a),
$$

where we take

$$
v(a):=-\frac{1}{b-a} \int_{a}^{b}\left(\int_{a}^{t}\left(f\left(\tau, \int_{a}^{\tau} v(s) \mathrm{d} s+D_{v}\right)+h(\tau)\right) \mathrm{d} \tau\right) \mathrm{d} t,
$$

and $D_{v}$ is the unique zero of $F_{v}$, because in this case, it is clear that $\int_{a}^{b} v(t)$ $\mathrm{d} t=0$. Thus, we consider the operator $T: \mathcal{C}([a, b], \mathbb{B}) \rightarrow \mathcal{C}([a, b], \mathbb{B})$, given by

$$
T(v)(t):=\int_{a}^{t} f\left(\tau, \int_{a}^{\tau} v(s) \mathrm{d} s+D_{v}\right) \mathrm{d} \tau+\int_{a}^{t} h(\tau) \mathrm{d} \tau+v(a),
$$

and, therefore, to find a fixed point for the operator $T$ is equivalent to find a solution of Eq. (3.6).

Step 3. Let us see that $T$ is continuous. Let $\left\{v_{n}\right\}$ be a sequence in $\mathcal{C}([a, b], \mathbb{B})$ that converges strongly to $v \in \mathcal{C}([a, b], \mathbb{B})$. Note that $\left\{D_{v_{n}}\right\}$ is a bounded sequence, and since $\mathbb{B}$ is a reflexive Banach space, we may assume that $D_{v_{n}} \rightarrow$ $D$. We can argue as in proof of Lemma 3.1 where now we can define $g_{n}(t):=$ $f\left(t, \int_{a}^{t} v_{n}(s) \mathrm{d} s+D_{v_{n}}\right)$ and $g(t)=f\left(t, \int_{a}^{t} v(s) \mathrm{d} s+D\right)$. The uniqueness of the zeros for $F_{v}$ allows us to conclude that $D_{v}=D$.

On the other hand, for each $t \in[a, b]$

$$
\begin{aligned}
\left\|T\left(v_{n}\right)(t)-T(v)(t)\right\| & \leq \int_{a}^{t}\left\|g_{n}(\tau)-g(\tau)\right\| \mathrm{d} \tau+\left\|v_{n}(a)-v(a)\right\| \\
& \leq \int_{a}^{b}\left\|g_{n}(\tau)-g(\tau)\right\| \mathrm{d} \tau+\left\|v_{n}(a)-v(a)\right\| \\
& \leq 2 \int_{a}^{b}\left\|g_{n}(\tau)-g(\tau)\right\| \mathrm{d} \tau,
\end{aligned}
$$

for all $n \in \mathbb{N}$. Then, $\left\|T\left(v_{n}\right)-T(v)\right\|_{\infty} \leq 2 \int_{a}^{b}\left\|g_{n}(\tau)-g(\tau)\right\| \mathrm{d} \tau$, for all $n \in \mathbb{N}$.

Since $f$ is sequentially weak-strong continuous, $\left\|g_{n}(\tau)-g(\tau)\right\| \rightarrow 0$ as $n \rightarrow \infty$ for all $\tau \in[a, b]$ and, furthermore, $\left\|g_{n}(t)\right\| \leq K$ for all $t \in[a, b]$ where $K \geq 0$ is the constant of boundedness of $f\left([a, b] \times B_{2(b-a)+\rho}\right)$ and $\rho>0$ satisfying $\left\|v_{n}\right\|_{\infty} \leq \rho$ for all $n \in \mathbb{N}$, because $\left\{v_{n}\right\}$ is a convergent sequence. Then, by the dominated convergence theorem, we obtain $T\left(v_{n}\right) \rightarrow T(v)$ as $n \rightarrow \infty$, which means that $T$ is continuous.

Step 4. We next prove that $T$ is compact. Let $C$ be a bounded subset of $\mathcal{C}([a, b], \mathbb{B})$ and let us see that $\overline{T(C)}$ is compact. To prove this, we shall make use of the Ascoli-Arzelà Theorem.

In the first place, let $0<\varrho=\varrho(C):=\sup \left\{\|v\|_{\infty}: v \in C\right\}<\infty$, because $C$ is bounded. Then, for any $v \in C$, we get to $\left\|\int_{a}^{\tau} v(s) \mathrm{d} s+D_{v}\right\| \leq$ 
$(b-a) \varrho+\delta_{(b-a) \varrho}$ for all $\tau \in[a, b]$, where $\delta_{(b-a) \varrho}$ is the constant given in (3.2). Since $f$ is sequentially weak-strong continuous, there exists $K>0$, such that $\|f(t, x)\| \leq K$ for all $t \in[a, b]$ and $x \in B_{R}[0]$, where $R:=(b-a) \varrho+\delta_{(b-a) \varrho}$. Then, for any $t \in[a, b]$ and $v \in C$, we have that

$$
\begin{aligned}
\|T(v)(t)\| & \leq 2 \int_{a}^{b}\left(\left\|f\left(\tau, \int_{a}^{\tau} v(s) \mathrm{d} s+D_{v}\right)\right\|+\|h(\tau)\|\right) \mathrm{d} \tau \\
& \leq 2(b-a)\left(K+\|h\|_{\infty}\right),
\end{aligned}
$$

that is, $(i)$ holds. On the other hand, for any $\varepsilon>0$, if we take $\delta(\varepsilon)=$ $\varepsilon /\left(K+\|h\|_{\infty}\right)>0$, then, for all $v \in C$ and $t, s \in[a, b]$ with $t>s$ and $|t-s| \leq \delta(\varepsilon)$, we obtain that

$$
\begin{aligned}
\|T(v)(t)-T(v)(s)\| & \leq \int_{s}^{t}\left(\left\|f\left(\tau, \int_{a}^{\tau} v(s) \mathrm{d} s+D_{v}\right)\right\|+\|h(\tau)\|\right) \mathrm{d} \tau \\
& \leq(t-s)\left(K+\|h\|_{\infty}\right)<\varepsilon,
\end{aligned}
$$

which means that $T(C)$ is equi-continuous. Finally, let us see that, for each $t \in[a, b]$, the set $\{T(v)(t): v \in C\}$ is compact in $\mathbb{B}$. Fix $v \in C$. For each $t \in[a, b]$, we have that

$$
\int_{a}^{t} f\left(\tau, \int_{a}^{\tau} v(s) \mathrm{d} s+D_{v}\right) \mathrm{d} \tau \in(t-a) \overline{\operatorname{conv}}\left(f\left([0, t] \times B_{R}[0]\right)\right) .
$$

Since $\mathbb{B}$ is reflexive, $[0, t] \times B_{R}[0]$ is weak compact and, since $f$ is sequentially weak-strong continuous, $f\left([0, t] \times B_{R}[0]\right)$ is compact. By Mazur's Compactness Theorem, we obtain that $(t-a) \overline{\operatorname{conv}}\left(f\left([0, t] \times B_{R}[0]\right)\right)$ is compact, for any $t \in[a, b]$.

Therefore, from Ascoli-Arzelà Theorem, we deduce that $T$ is a compact operator.

Step 5. Let us prove that $T$ has a fixed point in $\mathcal{C}([a, b], \mathbb{B})$. It is easy to see that, for any $v \in \mathcal{C}([a, b], \mathbb{B})$

$$
\|T(v)(t)\| \leq 2 \int_{a}^{b}\left\|f\left(\tau, \int_{a}^{t} v(s) \mathrm{d} s+D_{v}\right)\right\| \mathrm{d} \tau+2(b-a)\|h\|_{\infty} \quad \forall t \in[a, b] .
$$

(a) Suppose that $f$ is bounded. Then, there exists $\kappa \geq 0$, such that $\|f(t, x)\| \leq \kappa$ for all $(t, x) \in[a, b] \times \mathbb{B}$. Bearing in mind (3.7), one can prove that $T(\mathbb{B}) \subseteq B_{S}[0]$, where $S:=2(b-a)\left(\kappa+\|h\|_{\infty}\right)$. Thus, using Schauder's Fixed Point Theorem, we can ensure that $\left.T\right|_{B_{S}[0]}$ : $B_{S}[0] \rightarrow B_{S}[0]$ has a fixed point.

(b) Suppose that $\left(A_{2}\right)$ holds. Let us see that $T$ satisfies the LeraySchauder boundary condition on some closed ball. From (3.7) and $\left(A_{2}\right)$, for any $v \in \mathcal{C}([a, b], \mathbb{B})$

$$
\begin{aligned}
\|T(v)\|_{\infty} & \leq 2(b-a)\left(\Gamma\left((b-a)\|v\|_{\infty}+\left\|D_{v}\right\|\right)+\|h\|_{\infty}\right) \\
& \leq 2(b-a)\left(\Gamma\left((b-a)\|v\|_{\infty}+\delta_{(b-a)\|v\|_{\infty}}\right)+\|h\|_{\infty}\right) .
\end{aligned}
$$


From the hypothesis of $\Gamma$, we deduce that there exists $S>0$ such that, for all $\|v\|_{\infty} \geq S$

$$
\begin{aligned}
\frac{\|T(v)\|_{\infty}}{\|v\|_{\infty}} \leq & 2(b-a)^{2} \frac{\Gamma\left((b-a)\|v\|_{\infty}+\delta_{(b-a)\|v\|_{\infty}}\right)}{(b-a)\|v\|_{\infty}} \\
& +2(b-a) \frac{\|h\|_{\infty}}{\|v\|_{\infty}} \\
& <1 .
\end{aligned}
$$

Then, the Leray-Schauder boundary condition on the ball $B_{S}[0]$ holds. Therefore, by Leray-Schauder alternative Theorem, there exists at least a fixed point of $T$.

Remark 3.3. The method of proof is relying on fixed point theorems of LeraySchauder type, which does not yield uniqueness. It is an open question to ensure that the solution is unique or the possibility of multiple solutions under the assumptions of our main theorem. However, in the absence of condition (3.2), the existence of any solution cannot be guaranteed (see example 4.2 below). On the other hand, if conditions $\left(A_{1}\right)$ and $\left(A_{2}\right)$ fail, we cannot claim the uniqueness of solutions: for example, the following secondorder differential equation has multiple solutions:

$$
\left\{\begin{array}{l}
u^{\prime \prime}(t)=-u(t) \\
u(0)-u(2 \pi)=u^{\prime}(0)-u^{\prime}(2 \pi)=0 .
\end{array}\right.
$$

Finally, we point out that the hypothesis $\int_{a}^{b} h(t) \mathrm{d} t=0$ cannot be removed in our main result (see Example 4.2 below).

\section{Examples}

In this section, we present three examples of application of Theorem 3.1. Two of them for a system of $n(n \in \mathbb{N})$ second-order differential equations and the last one for a partial differential equation.

Example 4.1. If $h_{1}, \ldots, h_{n}:[a, b] \rightarrow \mathbb{R}$ are continuous functions with $\int_{a}^{b} h_{i}(t) \mathrm{d} t=0$, then the problem

$$
\left\{\begin{aligned}
u_{1}^{\prime \prime}(t) & =\frac{u_{1}(t)}{1+\left|u_{1}(t)\right|+\cdots+\left|u_{n}(t)\right|}+h_{1}(t) \\
\vdots & \\
u_{n}^{\prime \prime}(t) & =\frac{u_{n}(t)}{1+\left|u_{1}(t)\right|+\cdots+\left|u_{n}(t)\right|}+h_{n}(t) \\
u_{1}(a) & =u_{1}(b), \ldots, u_{n}(a)=u_{n}(b), \\
u_{n}^{\prime}(a) & =u_{n}^{\prime}(b), \ldots, u_{n}^{\prime}(a)=u_{n}^{\prime}(b),
\end{aligned}\right.
$$


has at least a solution in $\mathcal{C}^{2}\left([a, b], \mathbb{R}^{n}\right)$. Indeed, just to note that (4.1) can be rewritten as Problem $(1.1)$ where $h:=\left(h_{1}, \ldots, h_{n}\right), u=\left(u_{1}, \ldots, u_{n}\right)$ and $f: \mathbb{R}^{n} \rightarrow \mathbb{R}^{n}$ is given by $f(x)=\frac{x}{1+\|x\|_{1}}$. Here, $\|\cdot\|_{1}$ denotes the 1 -norm of $\mathbb{R}^{n}$.

It is clear that $f$ is bounded and weak-strong continuous. On the other hand, for each $M>0$, taking $\delta_{M}=M$, we have that $\langle f(y+x), x\rangle_{-} \geq 0$ for all $y \in B_{M}[0]$ and $\|x\|_{1} \geq \delta_{M}$. Indeed, fix $y \in B_{M}[0]$ and $\|x\|_{1} \geq \delta_{M}$, for any $j \in J(x)$

$$
\begin{aligned}
\langle f(y+x), j\rangle & =\frac{1}{1+\|y+x\|_{1}}\langle y+x, j\rangle=\frac{1}{1+\|y+x\|_{1}}\left(\|x\|_{1}^{2}+\langle y, x\rangle\right) \\
& \geq \frac{1}{1+\|y+x\|_{1}}\left(\|x\|_{1}^{2}-\|x\|_{1}\|y\|_{1}\right) \\
& =\frac{1}{1+\|y+x\|_{1}}\left(\|x\|_{1}-\|y\|_{1}\right)\|x\|_{1} \geq 0
\end{aligned}
$$

since $\|y\|_{1} \leq M$ and $\|x\|_{1} \geq \delta_{M}=M$.

Furthermore, $f$ is strictly accretive, because, for all $x, y \in \mathbb{R}^{n}$ with $x \neq y$, let $j$ be an element of $J(x-y)$; then

$$
\begin{aligned}
\langle f(x)-f(y), j\rangle= & \frac{1}{\left(1+\|x\|_{1}\right)\left(1+\|y\|_{1}\right)}\left\langle x-y+x\|y\|_{1}-y\|x\|_{1}, j\right\rangle \\
= & \frac{1}{\left(1+\|x\|_{1}\right)\left(1+\|y\|_{1}\right)}\left(\|x-y\|_{1}^{2}\right. \\
& \left.+\left\langle(x-y)\|y\|_{1}+y\left(\|y\|_{1}-\|x\|_{1}\right), j\right\rangle\right) \\
\geq & \frac{\|x-y\|_{1}^{2}}{\left(1+\|x\|_{1}\right)\left(1+\|y\|_{1}\right)}>0 .
\end{aligned}
$$

By Theorem $3.1\left(A_{1}\right)$, we deduce that Problem (4.1) has at least one solution $u \in \mathcal{C}^{2}\left([a, b], \mathbb{R}^{n}\right)$.

Remark 4.1. By carefully checking the above example, we find that other kinds of systems of equations can be solved. Just consider another norm $\|\cdot\|$ in $\mathbb{R}^{n}$. For example, we can ensure the existence of at least a periodic solution of a system of nonlinear second-order differential equations of the following type:

$$
u^{\prime \prime}(t)=\frac{u(t)}{1+\|u(t)\|}+h(t) .
$$

The following example shows that in Theorem 3.1, neither the hypothesis $\int_{a}^{b} h(t) \mathrm{d} t=0$ nor condition (3.2) can be omitted.

Example 4.2. The following problem does not have solution:

$$
\left\{\begin{array}{l}
u^{\prime \prime}(t)=\frac{u(t)}{1+|u(t)|}+2 \\
u(0)=u(1) \\
u^{\prime}(0)=u^{\prime}(1)
\end{array}\right.
$$


Indeed, suppose that $u_{0}$ is a solution of (4.2), then if we call $v(t)=u_{0}^{\prime}(t)$, we have

$$
v^{\prime}(t)=\frac{\int_{0}^{t} v(s) \mathrm{d} s+u_{0}(0)}{1+\left|\int_{0}^{t} v(s) \mathrm{d} s+u_{0}(0)\right|}+2
$$

Then,

$$
0=u_{0}^{\prime}(1)-u_{0}^{\prime}(0)=v(1)-v(0)=\int_{0}^{1} \frac{\int_{0}^{t} v(s) \mathrm{d} s+u_{0}(0)}{1+\left|\int_{0}^{t} v(s) \mathrm{d} s+u_{0}(0)\right|} \mathrm{d} t+2,
$$

This means that

$$
\int_{0}^{1} \frac{\int_{0}^{t} v(s) \mathrm{d} s+u_{0}(0)}{1+\left|\int_{0}^{t} v(s) \mathrm{d} s+u_{0}(0)\right|} \mathrm{d} t=-2
$$

which is a contradiction.

On the other hand, it is not difficult to see that the function $f:[0,1] \times$ $\mathbb{R} \rightarrow \mathbb{R}$ defined by $f(t, x)=\frac{x}{1+|x|}$ is under the conditions of Remark 4.1; therefore, hypothesis $\int_{a}^{b} h(t) \mathrm{d} t=0$ cannot be omitted.

If we call $f(t, x)=\frac{x}{1+|x|}+2$ and $h \equiv 0$, we may say that in the main result, condition (3.2) cannot be removed.

We next give an example in the case $f$ is unbounded and, moreover, the inequality required for $\Gamma$ in assumption $\left(A_{2}\right)$ holds for any closed interval $[a, b]$.

Example 4.3. Let $h_{1}, \ldots, h_{n}:[a, b] \rightarrow \mathbb{R}$ be continuous functions with $\int_{a}^{b} h_{i}(t) \mathrm{d} t=0$ and let $0<\alpha<1$. Then, the problem

$$
\left\{\begin{aligned}
u_{1}^{\prime \prime}(t) & =\frac{u_{1}(t) \sqrt{\left(u_{1}^{2}(t)+\cdots+u_{n}^{2}(t)\right)^{\alpha}}}{1+\sqrt{u_{1}^{2}(t)+\cdots+u_{n}^{2}(t)}}+h_{1}(t) \\
\vdots & \\
u_{n}^{\prime \prime}(t) & =\frac{u_{n}(t) \sqrt{\left(u_{1}^{2}(t)+\cdots+u_{n}^{2}(t)\right)^{\alpha}}}{1+\sqrt{u_{1}^{2}(t)+\cdots+u_{n}^{2}(t)}}+h_{n}(t) \\
u_{1}(a) & =u_{1}(b), \ldots, u_{n}(a)=u_{n}(b), \\
u_{1}^{\prime}(a) & =u_{1}^{\prime}(b), \ldots, u_{n}^{\prime}(a)=u_{n}^{\prime}(b)
\end{aligned}\right.
$$

has at least a solution in $\mathcal{C}^{2}\left([a, b], \mathbb{R}^{n}\right)$. This problem can be rewritten as Problem (1.1), where $h:=\left(h_{1}, \ldots, h_{n}\right), u=\left(u_{1}, \ldots, u_{n}\right)$ and $f: \mathbb{R}^{n} \rightarrow \mathbb{R}^{n}$ is given by $f(x)=\frac{x\|x\|_{2}^{\alpha}}{1+\|x\|_{2}}$. Here, $\|\cdot\|_{2}$ denotes the Euclidean norm of $\mathbb{R}^{n}$.

It is immediate that $f$ weak-strong continuous, because $\mathbb{R}^{n}$ is finite dimensional and $f$ is continuous. We claim that $f$ satisfies (3.2). Indeed, for each $M>0$, taking $\delta_{M}=M>0$, we deduce that

$$
\langle f(y+x), x\rangle=\frac{\|y+x\|_{2}^{\alpha}}{1+\|y+x\|_{2}}\left(\|x\|_{2}^{2}+\langle y, x\rangle\right)
$$




$$
\geq \frac{\|y+x\|_{2}^{\alpha}\|x\|_{2}}{1+\|y+x\|_{2}}\left(\|x\|_{2}-\|y\|_{2}\right) \geq 0
$$

for all $x, y \in \mathbb{R}^{n}$, with $\|y\|_{2} \leq M$ and $\|x\|_{2} \geq \delta_{M}=M$.

We now prove that $f$ is strictly accretive. Let $x, y \in \mathbb{R}^{n}$. Note that

$$
\begin{aligned}
\langle f(x)-f(y), x-y\rangle= & \frac{1}{\left(1+\|x\|_{2}\right)\left(1+\|y\|_{2}\right)}\left\langle\|x\|_{2}^{\alpha}\left(1+\|y\|_{2}\right) x\right. \\
& \left.-\|y\|_{2}^{\alpha}\left(1+\|x\|_{2}\right) y, x-y\right\rangle \\
= & \frac{1}{\left(1+\|x\|_{2}\right)\left(1+\|y\|_{2}\right)}\left(\left\langle\|x\|_{2}^{\alpha} x-\|y\|_{2}^{\alpha} y, x-y\right\rangle\right. \\
& \left.+\left\langle\|x\|_{2}^{\alpha}\|y\|_{2} x-\|y\|_{2}^{\alpha}\|x\|_{2} y, x-y\right\rangle\right) .
\end{aligned}
$$

In [11, Proof of Lemma 1], the authors prove that (in general, for $\alpha \geq 0$ )

$$
\left\langle\|x\|_{2}^{\alpha} x-\|y\|_{2}^{\alpha} y, x-y\right\rangle \geq \frac{1}{2}\|x-y\|_{2}^{2}\left(\|x\|_{2}^{\alpha}+\|y\|_{2}^{\alpha}\right) .
$$

On the other hand, if $\|y\|_{2}>\|x\|_{2}$, from Cauchy-Bunyakovsky-Schwarz inequality

$$
\begin{aligned}
& \left\langle\|x\|_{2}^{\alpha}\|y\|_{2} x-\|y\|_{2}^{\alpha}\|x\|_{2} y, x-y\right\rangle \\
& \quad=\left\langle\|x\|_{2}^{\alpha}\|y\|_{2}(x-y)+\left(\|x\|_{2}^{\alpha}\|y\|_{2}-\|y\|_{2}^{\alpha}\|x\|_{2}\right) y, x-y\right\rangle \\
& \quad=\|x\|_{2}^{\alpha}\|y\|_{2}\|x-y\|_{2}^{2}+\left(\|x\|_{2}^{\alpha}\|y\|_{2}-\|y\|_{2}^{\alpha}\|x\|_{2}\right)\langle y, x-y\rangle \\
& \quad=\|x\|_{2}^{\alpha}\|y\|_{2}\|x-y\|_{2}^{2}+\|x\|_{2}^{\alpha}\|y\|_{2}^{\alpha}\left(\|y\|_{2}^{1-\alpha}-\|x\|_{2}^{1-\alpha}\right)\langle y, x-y\rangle \\
& \quad \geq\|x\|_{2}^{\alpha}\|y\|_{2}\|x-y\|_{2}^{2}-\|x\|_{2}^{\alpha}\|y\|_{2}^{\alpha}\left(\|y\|_{2}^{1-\alpha}-\|x\|_{2}^{1-\alpha}\right)\|y\|_{2}\|x-y\|_{2} \\
& \quad=\|x\|_{2}^{\alpha}\|y\|_{2}\|x-y\|_{2}\left(\|x-y\|_{2}-\|y\|_{2}^{\alpha}\left(\|y\|_{2}^{1-\alpha}-\|x\|_{2}^{1-\alpha}\right)\right) \\
& \quad=\|x\|_{2}^{\alpha}\|y\|_{2}\|x-y\|_{2}\left(\|x-y\|_{2}-\|y\|_{2}+\|y\|_{2}^{\alpha}\|x\|_{2}^{1-\alpha}\right) \\
& \quad \geq\|x\|_{2}^{\alpha}\|y\|_{2}\|x-y\|_{2}\left(\|x-y\|_{2}-\|y\|_{2}+\|x\|_{2}\right) \geq 0 .
\end{aligned}
$$

Therefore, $\langle f(x)-f(y), x-y\rangle>0$ for all $x, y \in \mathbb{R}^{n}$ with $\|y\|_{2}>\|x\|_{2}$. This one, together with the symmetry of scalar product, implies that the above inequality holds for all $x, y \in \mathbb{R}^{n}, x \neq y$, that is, $f$ is strictly accretive.

Finally, let us see that $\left(A_{2}\right)$ holds. Indeed, just take the function $\Gamma$ : $[0, \infty) \rightarrow[0, \infty)$ defined by $\Gamma(s)=s^{\alpha+1} /(1+s)$. In this case, we have that $\|f(t, x)\|_{2} \leq \Gamma\left(\|x\|_{2}\right)$ for all $(t, x) \in[a, b] \times \mathbb{R}^{n}$ and moreover

$$
\lim _{s \rightarrow \infty} \frac{\Gamma\left(s+\delta_{s}\right)}{s}=\lim _{s \rightarrow \infty} \frac{\Gamma(2 s)}{s}=\lim _{s \rightarrow \infty} \frac{2^{\alpha}}{s^{1-\alpha}}=0<\frac{1}{2(b-a)^{2}},
$$

since $\delta_{s}=s$ is the constant given in (3.2) in this case, and $0<\alpha<1$. 
Example 4.4. Let $\Omega$ be an open convex and bounded subset of $\mathbb{R}^{n}$ and consider $\rho:[a, b] \times \Omega \rightarrow \mathbb{R}$ a function in $\mathcal{C}\left([a, b], L^{2}(\Omega)\right)$.

$$
\left\{\begin{array}{l}
\sum_{i=1}^{n} \frac{\partial^{2}}{\partial x_{i}^{2}}\left(\frac{\partial^{2} \phi}{\partial t^{2}}(t, x)-\rho(t, x)\right)=\phi(t, x) \text { in }(a, b) \times \Omega, \\
\phi(a, x)-\phi(b, x)=\frac{\partial \phi}{\partial t}(a, x)-\frac{\partial \phi}{\partial t}(b, x)=0 \text { in } \Omega, \\
\frac{\partial^{2} \phi}{\partial t^{2}}(t, x)-\rho(t, x)=0 \text { on }(a, b) \times \partial \Omega .
\end{array}\right.
$$

Definition 4.1. A function $\phi$ is said to be a strong solution of Problem (4.4) if the following assumptions are satisfied:

(i) $\phi \in \mathcal{C}^{2}\left([a, b], L^{2}(\Omega)\right)$,

(ii) $\frac{\partial^{2} \phi}{\partial t^{2}}(t, \cdot) \in W_{0}^{1,2}(\Omega) \cap W^{2,2}(\Omega)$ for all $t \in[a, b]$,

(iii) $\phi$ satisfies Eq. (4.4) in the sense of distributions over $\Omega$.

To do this study using the above results, consider the operator $\Delta$ : $L^{2}(\Omega) \rightarrow L^{2}(\Omega)$ with domain $D(\Delta):=W_{0}^{1,2}(\Omega) \cap W^{2,2}(\Omega)$ and, given $y \in$ $D(\Delta)$, define $\Delta y=\sum_{i=1}^{n} \frac{\partial^{2}}{\partial x_{i}^{2}} y(x)$, where the partial derivatives are taken in the sense of distributions over $\Omega$. Therefore, if we call $u(t):=\phi(t, \cdot) \in$ $L^{2}(\Omega)$ and $h(t):=\rho(t, \cdot) \in L^{2}(\Omega)$. We interpret and rewrite Problem (4.4) as follows:

$$
\left\{\begin{array}{l}
\Delta\left(u^{\prime \prime}(t)-h(t)\right)=u(t) \\
u(a)-u(b)=u^{\prime}(a)-u^{\prime}(b)=0 .
\end{array}\right.
$$

Our idea is to show that there exists at least $u \in \mathcal{C}^{2}\left([a, b] ; L^{2}(\Omega)\right)$ which is a solution of (4.5). To get it, we are going to assume that $h \in \mathcal{C}\left([a, b], L^{2}(\Omega)\right)$ and, moreover, $\int_{a}^{b} h(t) \mathrm{d} t=0$.

It is well known (for instance, see [2]) that $\Delta$ is an $m$-dissipative operator in $L^{2}(\Omega)$. Moreover, using Green's formula, we obtain that for $u \in D(\Delta)$

$$
\langle\Delta u, u\rangle=-\int_{\Omega}\langle\nabla u, \nabla u\rangle=-\|\nabla u\|_{2}^{2} ;
$$

this implies, by Poincaré's inequality (see Proposition 2.2), that

$$
\|\Delta u\|_{2}\|u\|_{2} \geq\|\nabla u\|_{2}^{2} \geq \frac{1}{c}\|\nabla u\|_{2}\|u\|_{2}
$$

and, bearing in mind (2.2), we obtain that

$$
\frac{1}{c \sqrt{c^{2}+1}}\|u\|_{1,2} \leq\|\Delta u\|_{2} \quad \text { for all } u \in D(\Delta) .
$$

The inequality given in (4.6) says that $\Delta$ is an $m$ - $\psi$-expansive dissipative operator (it is enough to take $\psi(t)=t /\left(\boldsymbol{c} \sqrt{\boldsymbol{c}^{2}+1}\right)$ ). Then, Remark 4.12 in [9] guarantees that there exists $\Delta^{-1}: L^{2}(\Omega) \rightarrow D(\Delta)$ and it is a strictly $m$-dissipative operator in $L^{2}(\Omega)$. This fact allows us to rewrite Problem (4.5) as follows:

$$
\left\{\begin{array}{l}
u^{\prime \prime}(t)=\Delta^{-1}(u(t))+h(t) \\
u(a)-u(b)=u^{\prime}(a)-u^{\prime}(b)=0 .
\end{array}\right.
$$


Finally, to show that Problem (4.7) admits at least a solution, we only have to check that $\Delta^{-1}: L^{2}(\Omega) \rightarrow D(\Delta)$ satisfies the conditions of Theorem 3.1.

First, let us see that $\Delta^{-1}: L^{2}(\Omega) \rightarrow D(\Delta)$ is sequentially weak-strong continuous. Indeed, from inequality (4.6), it is clear that

$$
\left\|\Delta^{-1} u\right\|_{2} \leq\left\|\Delta^{-1} u\right\|_{1,2} \leq \boldsymbol{c} \sqrt{c^{2}+1}\|u\|_{2},
$$

which means that $\Delta^{-1}: L^{2}(\Omega) \rightarrow D(\Delta)$ is continuous, because it is linear, then this operator is also weak-weak-continuous. On the other hand, if $M$ is a bounded subset of $L^{2}(\Omega)$, from (4.8), we have that $\Delta^{-1}(M)$ is a bounded subset of $W^{1,2}(\Omega)$; since the embedding $W^{1,2}(\Omega) \hookrightarrow L^{2}(\Omega)$ is compact (Rellich-Kondrachov's theorem), we have that $\Delta^{-1}(M)$ relatively compact in $L^{2}(\Omega)$; since $\Delta^{-1}$ is continuous, we may conclude that $\Delta$ is a weak-strong continuous map.

On the other hand, let us see that $\Delta^{-1}: L^{2}(\Omega) \rightarrow D(\Delta)$ satisfies Condition (3.3). Indeed, let $M>0$ and consider $g \in B_{M}[0] \subseteq L^{2}(\Omega)$; we have to check that $\left\langle\Delta^{-1}(u+g), u\right\rangle=\left\langle\Delta^{-1} u, u\right\rangle+\left\langle\Delta^{-1}(g), u\right\rangle$.

If we call $z:=\Delta^{-1}(u) \in W_{0}^{1,2}(\Omega) \cap W^{2,2}(\Omega)$, we may consider that $\Delta z=u$; therefore, by using Green's formula, Cauchy-Bunyakovsky-Schwarz inequality, and (4.8), we derive that

$$
\begin{aligned}
\left\langle\Delta^{-1}(u+g), u\right\rangle & \leq-\|\nabla z\|_{2}^{2}+\left\|\Delta^{-1} g\right\|_{2}\|u\|_{2} \\
& \leq-\|\nabla z\|_{2}^{2}+c \sqrt{c^{2}+1}\|g\|_{2}\|u\|_{2} .
\end{aligned}
$$

This means that for every $g \in B_{M}[0]$

$$
\left\langle\Delta^{-1}(u+g), u\right\rangle \leq-\|\nabla z\|_{2}^{2}+c \sqrt{c^{2}+1} M\|u\|_{2} .
$$

Now, using (2.2), we get

$$
\left\langle\Delta^{-1}(u+g), u\right\rangle \leq-\frac{1}{\boldsymbol{c}^{2}+1}\|z\|_{1,2}^{2}+\boldsymbol{c} \sqrt{\boldsymbol{c}^{2}+1} M\|u\|_{2} .
$$

Finally, since it is clear that $\|\Delta z\|_{2} \leq\|\Delta z\|_{1,2} \leq\|z\|_{1,2}$, we conclude that

$$
\begin{aligned}
\left\langle\Delta^{-1}(u+g), u\right\rangle & \leq-\frac{1}{\boldsymbol{c}^{2}+1}\|u\|_{2}^{2}+\boldsymbol{c} \sqrt{\boldsymbol{c}^{2}+1} M\|u\|_{2} \\
& =\|u\|_{2}\left(\boldsymbol{c} \sqrt{\boldsymbol{c}^{2}+1} M-\frac{1}{\boldsymbol{c}^{2}+1}\|u\|_{2}\right) \leq 0,
\end{aligned}
$$

whenever $M \boldsymbol{c}\left(\boldsymbol{c}^{2}+1\right)^{3 / 2} \leq\|u\|_{2}$; hence, we may take $\delta_{M}:=M \boldsymbol{c}\left(\boldsymbol{c}^{2}+1\right)^{3 / 2}$.

Second, we have already seen that $\Delta^{-1}: L^{2}(\Omega) \rightarrow D(\Delta)$ is a strictly dissipative operator, and thus, it satisfies Condition (3.5).

Finally, if we define $\Gamma:[0, \infty) \rightarrow[0, \infty)$ by $\Gamma(s):=c \sqrt{c^{2}+1} s$. From (4.8), we have that $\left\|\Delta^{-1} u\right\|_{2} \leq \Gamma\left(\|u\|_{2}\right)$ for all $u \in L^{2}(\Omega)$. Thus

$$
\lim _{s \rightarrow \infty} \frac{\Gamma\left(s+\delta_{s}\right)}{s}=c \sqrt{c^{2}+1}+c^{2}\left(c^{2}+1\right)^{2} .
$$

Therefore, as a consequence of Theorem 3.1, under the assumption

$$
c \sqrt{c^{2}+1}+c^{2}\left(c^{2}+1\right)^{2}<\frac{1}{2(b-a)^{2}},
$$


where $\boldsymbol{c}=\boldsymbol{c}(\Omega)$ is the constant in the Poincaré's inequality (2.1), we have existence of periodic solutions of Problem (4.7), and hence the following:

Proposition 4.1. Let $\Omega$ be an open convex and bounded subset of $\mathbb{R}^{n}$ and $a, b \in \mathbb{R}$ satisfying (4.9). Let $\rho \in \mathcal{C}\left([a, b], L^{2}(\Omega)\right)$ with $\int_{a}^{b} \rho(t,.) \mathrm{d} t=0$. Then, Problem (4.4) has at least one strong solution.

Remark 4.2. The optimal constant $\boldsymbol{c}$ in the Poincaré inequality (Proposition 2.2) is sometimes known as the Poincaré constant for the domain $\Omega$. Determining the Poincaré constant is, in general, a very hard task that depends upon the value of $p$ and the geometry of the domain $\Omega$. Nevertheless, it is well known, see [15, Theorem 3], that if $\Omega:=\left\{(x, y) \in \mathbb{R}^{2}: x \in(0,1), 0<\right.$ $y<1-x\}$, then the Poincaré constant $\boldsymbol{c}=\boldsymbol{c}(\Omega, 2)=\frac{1}{\pi}$. Therefore, from Proposition 4.1, we may derive that Problem (4.4) on this domain $\Omega$ has at least one strong solution whenever $0<b-a \leq 1$.

\section{Acknowledgements}

The research of the second author has been partially supported by PGC2018098474-B-C21. The authors are grateful to Julián Toledo for his comments which improved the paper. Finally, we would like to thank the referees for their valuable recommendations.

Funding Open Access funding provided thanks to the CRUE-CSIC agreement with Springer Nature.

Open Access. This article is licensed under a Creative Commons Attribution 4.0 International License, which permits use, sharing, adaptation, distribution and reproduction in any medium or format, as long as you give appropriate credit to the original author(s) and the source, provide a link to the Creative Commons licence, and indicate if changes were made. The images or other third party material in this article are included in the article's Creative Commons licence, unless indicated otherwise in a credit line to the material. If material is not included in the article's Creative Commons licence and your intended use is not permitted by statutory regulation or exceeds the permitted use, you will need to obtain permission directly from the copyright holder. To view a copy of this licence, visit http:// creativecommons.org/licenses/by/4.0/.

Publisher's Note Springer Nature remains neutral with regard to jurisdictional claims in published maps and institutional affiliations.

\section{References}

[1] Ariza-Ruiz, D., Garcia-Falset, J., Reich, S.: The Bolzano-Poincaré-Miranda theorem in infinite-dimensional Banach spaces. J. Fixed Point Theory Appl. 21(2), Art. 59, 12 pp (2019)

[2] Barbu, V.: Nonlinear Differential Equations of Monotone Types in Banach Spaces. Springer, New York (2010) 
[3] Brezis, H.: Functional Analysis. Sobolev Spaces and Partial Differential Equations, Universitext, Springer, New York (2011)

[4] Brown, R.F.: A topological introduction to nonlinear analysis. Birkhäuser (1993)

[5] Cioranescu, I.: Geometry of Banach Spaces. Kluwer Academic Publishers, Dordrecht, Duality Mappings and Nonlinear Problems (1990)

[6] De Coster, C., Habets, P.: The lower and upper solutions method for boundary value problems. Handbook of Differential Equations, pp. 69-160. Elsevier/North-Holland, Amsterdam (2004)

[7] Edwards, R.E.: Functional Analysis. Holt, Rinehart and Winston (1965)

[8] Franco, D., Torres, P.J.: Periodic solutions of singular systems without the strong force condition. Proc. Am. Math. Soc. 136, 1229-1236 (2008)

[9] Garcia-Falset, J.: Existence of fixed points for the sum of two operators. Math. Nachr. 283, 1736-1757 (2010)

[10] Hakl, R., Torres, P.J., Zamora, M.: Periodic solutions of singular second order differential equations: upper and lower functions. Nonlinear. Anal. 74, 70787093 (2011)

[11] Hanebaly, E., Marzouki, B.: Periodic and almost periodic solutions for multivalued differential equations in Banach spacess. Electron. J. Differ. Equ. 2000(24), 1-16 (2000)

[12] Jiang, Y.: Periodic solutions of second-order non-autonomous dynamical systems with vanishing Green's functions. Electron. J. Differ. Equ. 2019(47), 1-12 (2019)

[13] Kannan, R., Ortega, R.: Periodic solutions of Pendulum-type equations. J. Differ. Equ. 59, 123-144 (1985)

[14] Keyantuo, V., Lizama, C.: Periodic solutions of second order differential equations in Banach spaces. Math. Z. 253, 489-514 (2016)

[15] Kikuchi, F., Liu, X.: Estimation of interpolation error constants for the $P_{0}$ and $P_{1}$ triangular finite elements. Comput. Methods Appl. Mech. Eng. 196, 3750-3758 (2007)

[16] Knobloch, H.W.: On the existence of periodic solutions for second order vector differential equations. J. Differ. Equ. 9, 67-85 (1971)

[17] Kryszewski, W., Siemianowshki, J.: The Bolzano mean-value theorem and partial differential equations. J. Math. Anal. Appl. 457, 1452-1477 (2018)

[18] Leoni, G.: A First Course in Sobolev Spaces, Graduate Studies in Mathematics, vol. 105. American Mathematical Society, Providence, RI (2009)

[19] Liu, J., Yu, W.: Two solutions to superlinear Hamiltonian systems with impulsive effects. Appl. Math. Lett. 102, 106162 (2020)

[20] Szymańska-Dȩbowska, K.: On a generalization of the Miranda theorem and its application to boundary value problems. J. Differ. Equ. 258, 2686-2700 (2015) 
David Ariza-Ruiz and Jesús Garcia-Falset

Departament d'Anàlisi Matemàtica

Universitat de València

Dr. Moliner 50

46100 Burjassot València

Spain

e-mail: david.ariza@uv.es

Jesús Garcia-Falset

e-mail: garciaf@uv.es

Received: August 28, 2020.

Revised: April 30, 2021.

Accepted: December 7, 2021. 